\title{
Jóvenes españoles entre dos siglos (1984-2017)
}

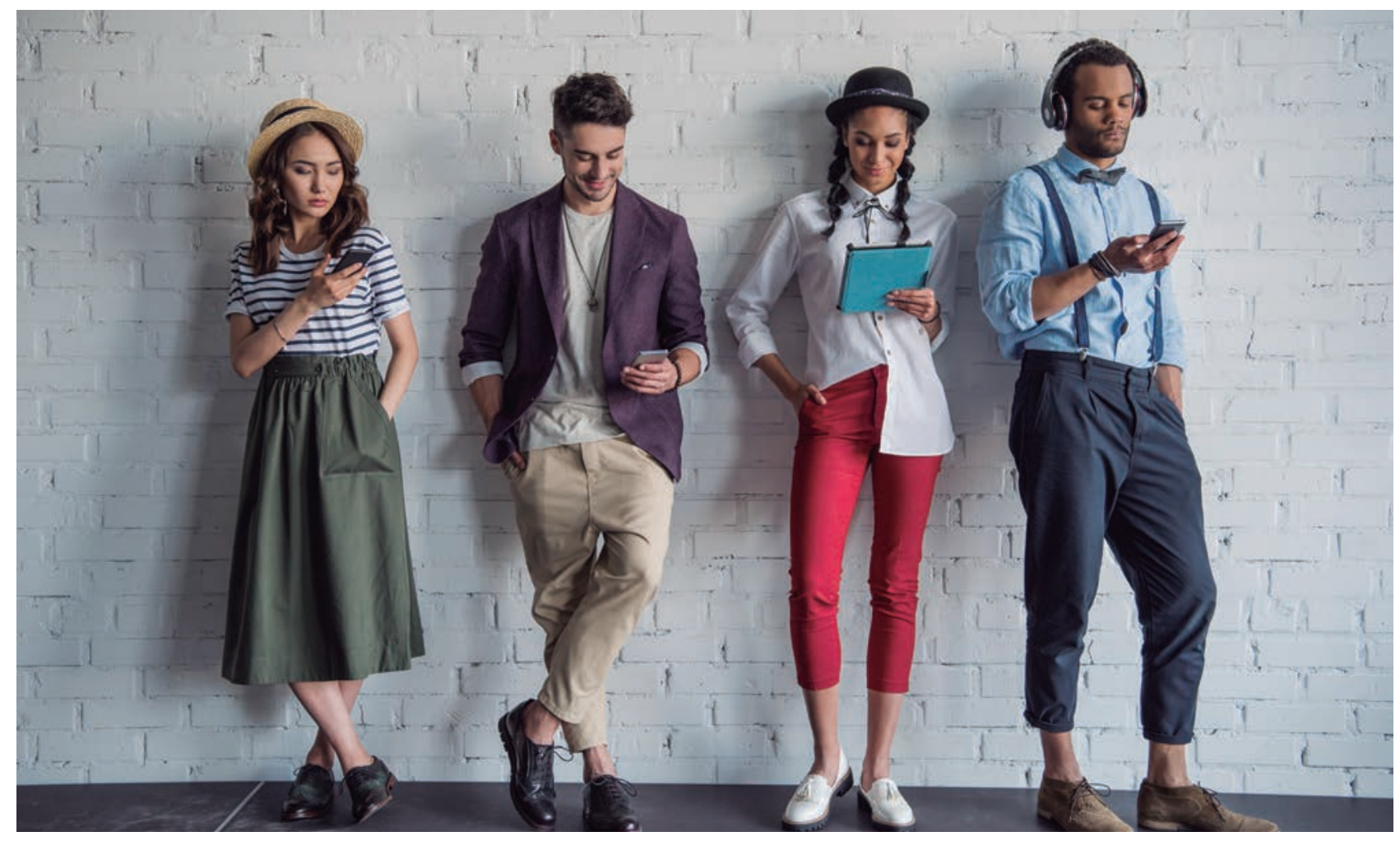

Este artículo ofrece una síntesis de los

principales hallazgos del último informe

sobre los jóvenes españoles de la Fundación

Santa María/Observatorio de la Juventud en

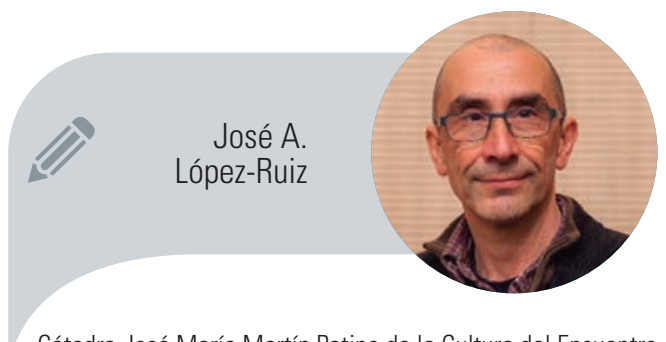

Cátedra José María Martín Patino de la Cultura del Encuentro Universidad Pontificia Comillas josealr@comillas.edu

Iberoamérica, publicado a finales del año

2017. Los datos de la última encuesta son

comparados con los de anteriores informes,

remontando en algunos casos hasta el primero

de ellos, que data del año 1984. 


\section{Introducción}

El informe Jóvenes Españoles entre dos siglos, publicado por la Fundación Santa María (en adelante Fundación Sm) se caracteriza por un especial interés en la serie histórica que arranca en el año 1984, aportando la perspectiva comparada de cerca de 40 años de estudios sociológicos. Los estudios de la Fundación Sm se distinguen por tener un especial interés en los valores y actitudes de los jóvenes, mientras que otras publicaciones, como por ejemplo las del Instituto de la Juventud (INJUVE 2016, 2015, 2010, entre otras publicaciones') o las del Centro Reina Sofía sobre Adolescencia y Juventud², se dirigen a estudiar cuestiones de tipo estructural como son empleo, salud, educación, sexualidad o vivienda, entre otras. Desde este encuadre, el artículo viene a ofrecer una "vista aérea" de los principales resultados del estudio, refiriéndose a los cinco capítulos en los que presenta el informe: los dos primeros tratan sobre los valores e integración sociopolítica, el tercero sobre familia y, el cuarto, sobre ocio y cultura juveniles. El quinto y último capítulo aborda las vivencias religiosas de los jóvenes.

El lector interesado en profundizar en el informe puede consultarlo o descar-

1 INJUVE 2016, Jorge Benedicto (Dir.) "Informe Juventud en España 2016", disponible en http:// www.injuve.es/sites/default/files/2017/24/publicaciones/informe-juventud-2016.pdf (acceso 9-10-18); 2015 "Sondeo de opinión y situación de la gente joven 2014 (3. ${ }^{2}$ encuesta)"; 2010 "Juventud en cifras: Ocio y tiempo libre" http://www.injuve.es/sites/default/files/JCifras-Ocio-Dic2010. pdf (acceso 9-10-18).

2 Ver Elena Rodríguez San Julián y Juan Carlos Ballesteros Guerra, 2013; "Crisis y contrato social: los jóvenes en la sociedad del futuro" Centro Reina Sofía sobre Adolescencia y Juventud, FAD; Alessandro Gentile, Anna Sanmartín Ortí, Ana Lucía Hernández Cordero, 2014 "La sombra de la crisis: la juventud española en el horizonte de 2018". Madrid: Centro Reina Sofía sobre Adolescencia y Juventud, FAD; Javier Elzo y Eusebio Megías (codirs.), 2014 "Jóvenes y valores: un ensayo de tipología" Madrid: Centro Reina Sofía sobre Adolescencia y Juventud, FAD, también entre otros muchos disponibles desde http:// www.adolescenciayjuventud.org/que-hacemos/ monografias-y-estudios.

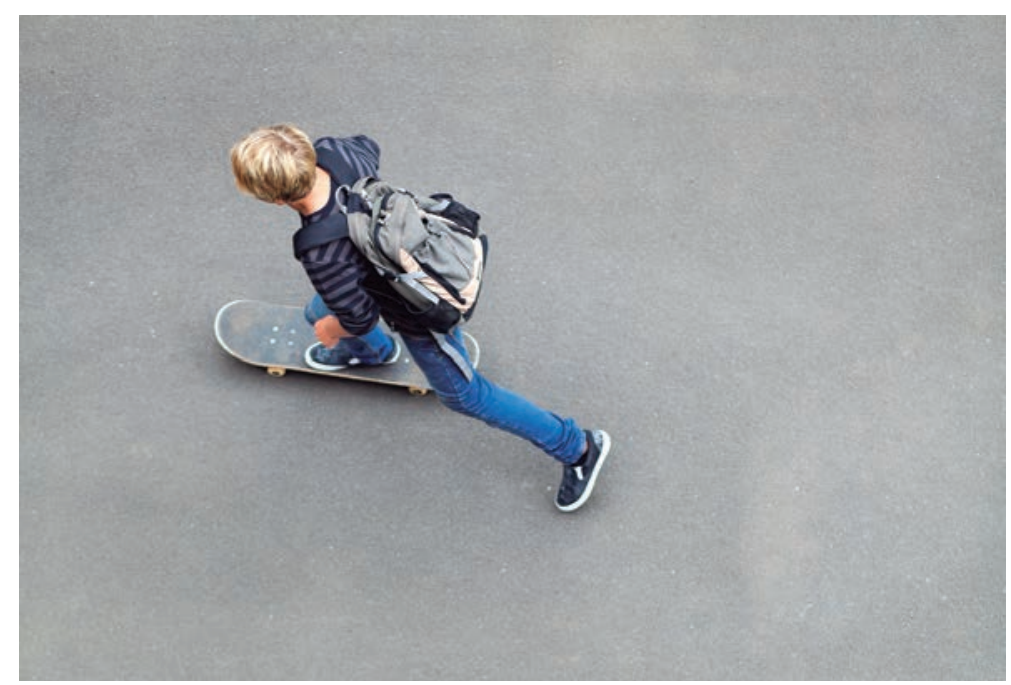

garlo íntegramente desde el Observatorio de los Jóvenes de la Fundación Sm (https://www.observatoriodelajuventud. org/jovenes-espanoles-entre-dos-siglos-1984-2017/).

\section{Valores morales y confianza en las instituciones}

En los valores socioculturales relacionados con la implicación y participación sociopolítica se han detectado tendencias que desmienten la idea de cambios bruscos generacionales, existen tendencias claras de desafección e inacción política detectadas ya en el primer informe de 1984.

Durante el periodo de crisis se produce una fuerte aceleración del laxismo moral que afecta a todos los comportamientos propuestos (justificación de comportamientos morales), un hecho sin parangón en los informes realizados desde el año 84.

Se ha producido un hundimiento de la confianza en las instituciones sociales solamente comparable al "gran hundimiento" entre 1999 y 2005, y que afecta a la mayoría de las instituciones. Los casos de la prensa, la monarquía y los sindicatos son perfectamente comparables con los grandes descalabros de aquella época.

\section{Integración sociopolítica}

No se ha producido una "brecha generacional" en lo que se refiere al autoposicionamiento político, actualmente un tema de gran calado debido al auge y cierto éxito entre los jóvenes de nuevas formaciones políticas.

Se ha detectado un mayor interés de los jóvenes en política, que contrasta con 


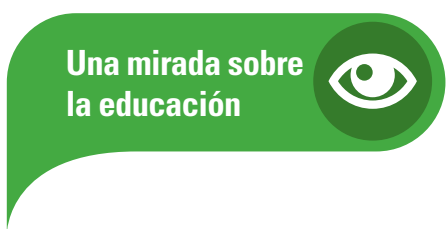

Gráfico 1. Opiniones sobre la política y los políticos 2005-2016

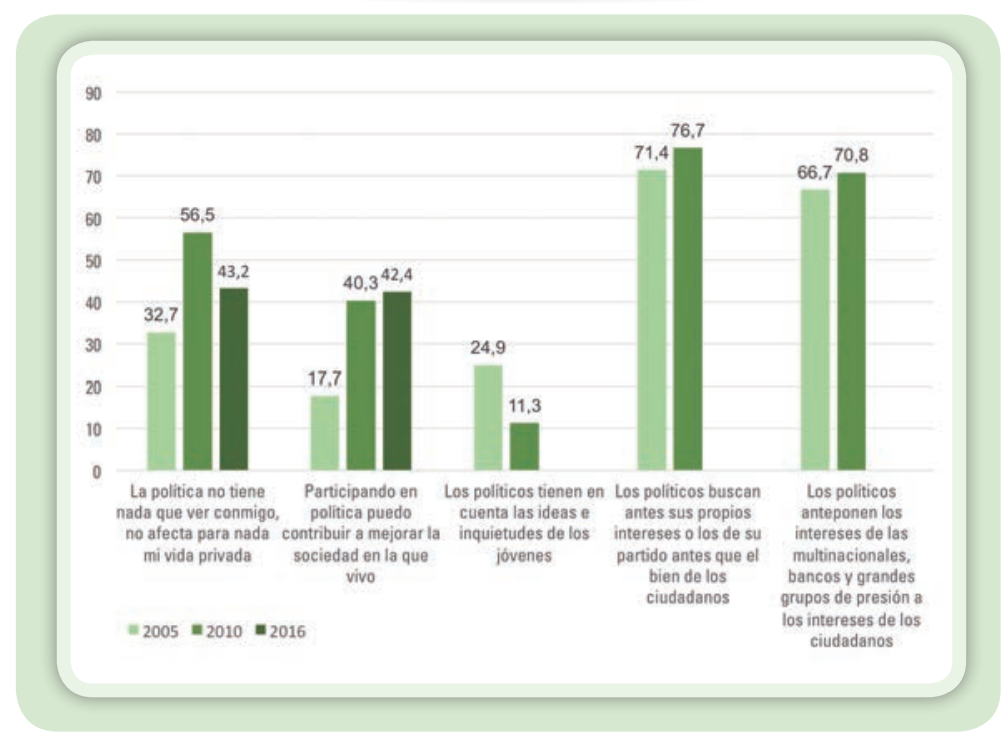

una falta de acción (y de perspectivas de acción). Una paradoja que puede indicar que se esté dando una cesión de la responsabilidad política a los partidos emergentes que mantiene intacta la pasividad de los jóvenes en la arena política.

Por otro lado, se da un cambio de tendencia importante en la identificación territorial de los jóvenes españoles con el año 1999 como inflexión, creciendo con fuerza la identificación con España, frente al crecimiento hasta este año de la región y comunidad autónoma.

El estudio sobre los valores democráticos básicos es una novedad de este informe, al retomar una pregunta de 1989, en comparación, hoy se considera menos democrático el Estado español, se considere menor la libertad de expresión (ambos valores cerca del aprobado raspado — del 5-) y menor el respeto por la ley y el orden y la tolerancia (ambos ya por debajo del 5).

\section{La centralidad de la familia para los jóvenes \\ Constante centralidad de la familia para los jóvenes}

La importancia relativa de la familia es muy alta y no ha descendido a lo largo de los años, actualmente el 97\% de los jóvenes la considera bastante o muy importante. Siendo la familia uno de los máximos referentes culturales para los jóvenes a la hora de entender el mundo de hoy y situarse dentro del mismo, la crisis incluso parece reforzar su valor.

Aunque los jóvenes viven rodeados de información, con redes de comunicación globalizadas de acceso cada vez más instantáneos y rápidos, la familia se sigue presentando como uno de los máximos referentes culturales para los jóvenes a la hora de entender el mundo de hoy y situarse dentro del mismo.

Actualmente la familia es para el $62 \%$ de los jóvenes el lugar donde más hablan de "las cosas importantes en cuanto a ideas e interpretaciones del mundo", citándola por encima de sus pares y amigos, e incluso por delante de los centros educativos, los libros o los medios de comunicación.

En el transcurso de una larga crisis económica y tras su paso, la familia se convierte en un agente social fundamental como mediador entre el impacto de la globalización, los recursos comunitarios - a menudo escasos - y las acciones del Estado - más o menos escasas y deficientes-y los individuos.

\section{Evolución de las formas de convivencia preferidas por los jóvenes}

La tercera parte de los jóvenes entrevistados manifiesta el deseo de casarse (33\%), si bien lo haría conviviendo antes con su pareja, mientras que otro tercio no sabe lo que haría (un 29\%). En los últimos años se observa un aumento en la indecisión e indefinición en cuanto a formas de convivencia deseadas para el futuro, más de 10 puntos porcentuales por encima de resultados anteriores.

Actualmente 1 de cada 3 jóvenes querría casarse conviviendo antes con su pareja, 1 de cada 4 ve el matrimonio como un "certificado burocrático-administrativo" innecesario y 1 de cada 10 piensa que es una mera "apariencia social".

No obstante, la tendencia a elegir el matrimonio tras una convivencia previa con la pareja ha ido disminuyendo progresivamente en estos años.

\section{Los límites de las reglas y la libertad en la familia}

Aunque el buen clima siga siendo un rasgo fundamental de la convivencia familiar, los jóvenes hoy discuten más que 
en años precedentes: entre los motivos de discusión destacan las cuestiones relativas a la colaboración en el trabajo doméstico (48\%), algo más de un 40\% discute también por asuntos referentes a los estudios y aumentan respecto a años anteriores las discusiones por dinero (39\%).

Disminuyen respecto al año 2010 las discusiones por la hora de llegada o por pasar la noche fuera, así como por cuestiones ideológico-políticas y referentes a la religión. En la última encuesta desciende de forma significativa la proporción de entrevistados que opina que los jóvenes españoles tienen un nivel de libertad "bastante adecuado": actualmente 1 de cada 3 jóvenes opina que tienen "más libertad de la que deberían, lo que supone un incremento del 12\% respecto a los jóvenes de finales de los años noventa.

\section{La educación dentro de la familia}

Para los jóvenes las cualidades que más pueden transmitir los padres en el hogar son cuatro: "buenos modales", "tolerancia y respeto por los demás", "sentido de la responsabilidad" y "honestidad". Estas cuatro cualidades eran también las más citadas en los años ochenta, aunque la más importante entre ellas, en 1984, fue el "sentido de la responsabilidad".

\section{Cultura y ocio juveniles Imagen social de los jóvenes}

A lo largo de la serie histórica, desde 1994, hay tres características básicas que sobresalen respecto al resto: Ios jóvenes se consideran "consumistas", "rebeldes" e "independientes", si bien la última de estas características ha ido perdiendo importancia paulatinamente (un 55\% de menciones en 1994 y solo un $21 \%$ en 2016).

Los resultados de 2017 caracterizan también a unos jóvenes "demasiado preocupados por la imagen" (39\%), algo "egoístas" (35\%), pero también en parte "indignados por la situación sociopolítica" (32\%).

Los rasgos en los que se constata un mayor incremento son "bajo sentido del deber" $(+13,4 \%$ respecto a 1994$)$, "egoístas" $(+12,7 \%)$ y "con poco sentido del sacrificio" (+11,4\%).
Gráfico 2. Características y autoimagen de los jóvenes

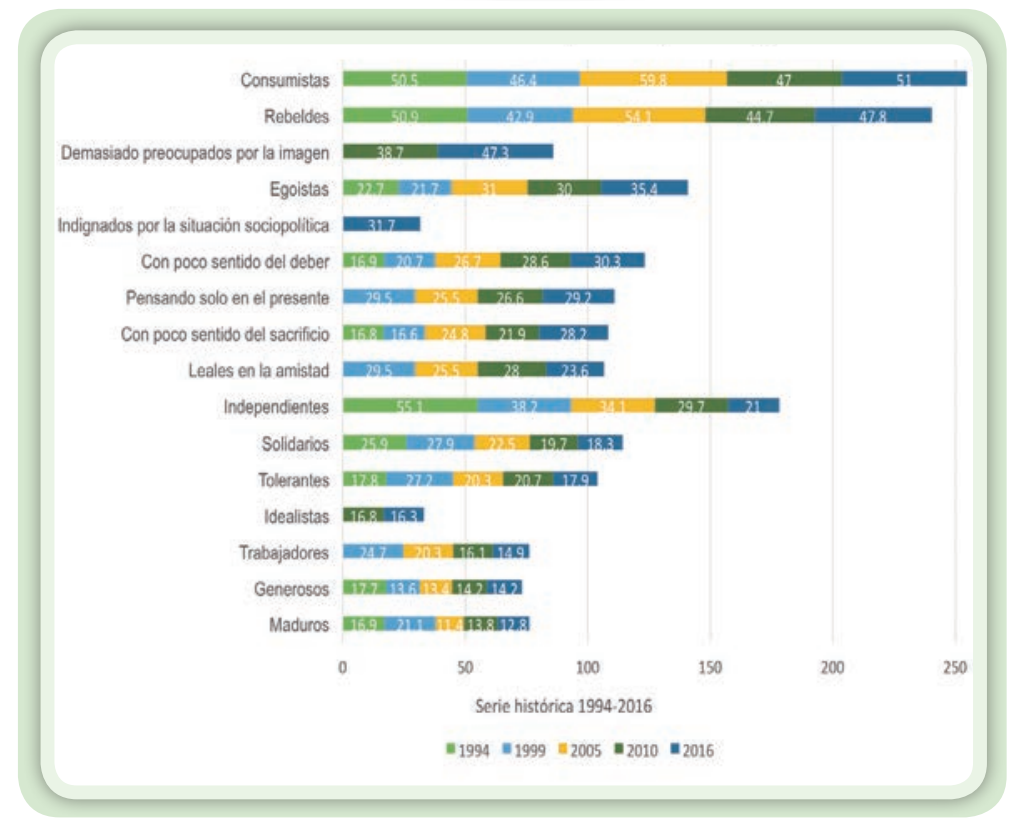

Cualidades positivas, como "leales en la amistad", "solidarios", "tolerantes" o "idealistas", entre otras, han disminuido en la identificación de los jóvenes, o se mantienen en niveles bajos (un 20\% de menciones o menos).

En resumen, la incidencia de la larga crisis económica que ha sufrido España, parece reflejarse en una menor independencia, menor sentido del deber y del sacrificio, en unos jóvenes que también se ven más egoístas que antes.

\section{Diversificación y generalización de las actividades de ocio}

El ocio ocupa un lugar central en la cultura juvenil: comparando con otros valores importantes, el valor que conceden los jóvenes al ocio es similar al que tienen las amistades, los estudios e incluso la formación y la competencia profesionales. Actualmente el ocio se configura más como un ocio asociado a múltiples actividades y menos como un ocio identificado con salir de noche los fines de semana, algo que era más habitual en años anteriores.

El abanico de actividades practicadas por los jóvenes es amplio y de práctica extendida, si bien es un dato notable que la mayoría de los jóvenes parecen hacer el mismo tipo de cosas y prácticamente en el mismo orden de preferencia; tan solo hay 5 de las 25 actividades en las que sean menos del 50\% los jóvenes que las practican. 
Gráfico 3. Evolución de las preferencias en ocio y tiempo libre

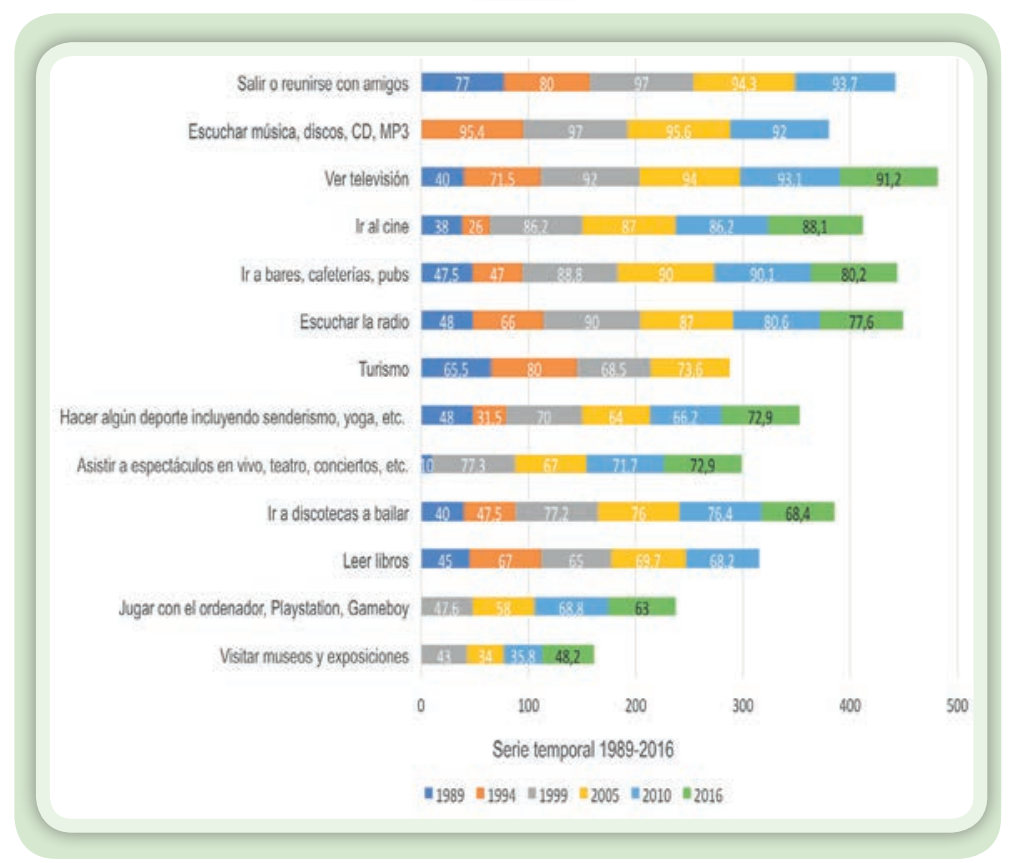

त En 2017, según la extensión de la práctica, se puede distinguir ciertas actividades de práctica totalmente generalizada (>85\%) ver películas o series (un 94,2\%), salir o reunirse con amigos (un 93,7\%), escuchar música, discos, CD, MP3 (un 92\%), ver televisión (un $91,2 \%$ ), ir al cine (un $88,1 \%$ ) y ver canales temáticos en internet y visualizar diferentes contenidos en YouTube $(86,8 \%)$.

Cambios destacados en la serie temporal: teniendo en cuenta la serie temporal más larga, se puede comprobar que se produce una clara generalización y diversificación de actividades, no solo por la proliferación de medios informáticos y audiovisuales, sino también por un incremento en actividades culturales, deporte, al lado de los clásicos "ocios de consumo", como salir a bares o discotecas, cine, viajes, compras, entre otros.

En este campo, la crisis económica no parece haber impedido una evolución del ocio impulsada por la "democratización" de la cultura digital, la accesibilidad de los viajes y de diferentes ámbitos culturales (teatro, cine, conciertos, etc.) "accesibles" aún para el reducido poder adquisitivo de la mayoría de los jóvenes, junto al incremento de la práctica de deporte.

\section{Lectura de libros y prensa ¿cuánto leen los nativos digitales?}

Aunque los jóvenes españoles, comparado con otros países europeos, leen poco, no leen menos que los jóvenes de unas décadas atrás. Los datos obtenidos entre los años 2005 y 2016 permiten confirmar que existe un aumento general de la lectura. Cerca de la tercera parte de los jóvenes entrevistados en el año 2016 declara que no lee ningún libro (un 31,7\%), un dato alarmante si se tiene en cuenta que la mayor parte de los jóvenes son estudiantes en diferentes niveles académicos y que, entre el resto, predominan los jóvenes con estudios secundarios y superiores.

En cuanto a lectura de prensa, algo más de la tercera parte de los jóvenes no realiza el seguimiento informativo de ningún tipo de periódico (un 35,9\%); un porcentaje elevado pero similar al de la población adulta (un 26,5\% según datos del Estudio General de Medios para 2016).

Al comparar los resultados del año 2016 con los informes anteriores, se observa que disminuye la lectura de periódicos desde 1999, probablemente como efecto del aumento del uso de internet.

\section{La extensión del uso de internet: un medio que llega para quedarse}

El acceso a internet y el uso de teléfonos móviles, uno de los principales medios de acceso a la red, pasa en pocos años a tener una valencia prácticamente absoluta: en 2017 el 99\% de los jóvenes ha utilizado internet en los últimos 4 meses.

Comparativamente, desde 1999 se produce un crecimiento exponencial, con un incremento del 58\% entre dicho año y 2005, otro 23\% en los siguientes años para llegar al 95\% de acceso en el año 2010 y alcanza prácticamente el 100\% actual.

Caracterización del uso de internet:

入 Entre las funciones y los usos que encuentran hoy día en internet los jóvenes, en 2016 se encuentra "buscar cosas en Google" y "ver vídeos en YouTube", que eran también los más citados en el informe anterior. Vemos cómo 
estas dos plataformas se convierten en las principales puertas de entrada a la red y el 93\% de los jóvenes las utilizan.

$\boldsymbol{\lambda}$ La siguiente utilidad más frecuente es "ver películas online o bajadas de la red", algo que hacen casi el $80 \%$ de los jóvenes, un 27,4\% más que en el año 2010, una actividad relacionada con "descargar películas" y "ver programas de TV", también muy generalizadas.

$\boldsymbol{\lambda}$ Se inicia con este informe el seguimiento de las formas de acoso y violencia que se producen a través de internet y las redes sociales, conocidas como ciberacoso y ciberbullying, en el colegio o centro educativo, por parte de amigos y conocidos, en este momento se registran incidencias en torno al $12 \%$ de los casos totales.

\section{Jóvenes y religión}

Entre los años 1994 y 2005 un 30\% de los jóvenes dejaron de definirse como católicos, entre 2010 y 2016 ha seguido descendiendo $(-13 \%)$, situando el porcentaje de católicos en menos de la mitad de los jóvenes (40\%).

Al mismo tiempo, aumentan el agnosticismo de un $7 \%$ a un $14 \%$ entre los años 2005 y 2017, y el porcentaje de jóvenes que se declaran ateos, que pasa de un $7 \%$ en 1994 a un 24\% en el actual informe.

En cuanto a la práctica religiosa, cerca del $80 \%$ de los jóvenes afirma no asistir nunca o prácticamente nunca a la iglesia, mientras que declaran ir una vez por semana el $7 \%$, con mayor frecuencia el $1 \%$ y una vez por mes el $5 \%$.

También entre 2005 y 2017 incluso se reduce la asistencia esporádica a la igle-

\section{HEMOS HABLADO DE}

\section{Jóvenes; valores; familia; cultura y ocio.}

Este artículo fue solicitado por PADRES Y MAESTROS en diciembre de 2018, revisado y aceptado en marzo de 2019. sia por Navidad y otras celebraciones puntuales.

\section{Consideraciones finales}

El principal interés del estudio Jóvenes españoles entre dos siglos (1984-2017) es interpretar los aconteceres y vivencias que resultan importantes para los jóvenes, dar a conocer sus posicionamientos valorativos y actitudinales, de modo que, resulta de especial interés para todo tipo de educadores y profesionales que trabajen con jóvenes, incluyendo pastoral, resulta también desde interés para las propias familias y padres.

Es importante leer e interpretar los datos que ofrece este informe en el contexto de una seria crisis económica, con años de estancamiento y recesión, una crisis que ha afectado a la mayoría de las familias y los jóvenes — dejando su huella y, en parte, sigue afectando- con un aumento del desempleo, la precariedad laboral e incrementando las desigualdades sociales. Pero también en el horizonte vital de los jóvenes están otras crisis, si es como telón de fondo o como espada de Damocles dependerá del futuro, como la crisis medioambiental, crisis de la educación, de la religión, de la política nacional e internacional, del matrimonio, y así podríamos seguir. Lo único que parece no estar en crisis es la crisis misma, aun así hay filósofos que dicen que los síntomas del malestar actual se traducen en el aburrimiento y el cansancio, algo que los jóvenes a veces parecen trasmitir o reflejar cuando se refieren al mundo adulto $•$

\section{(D.) DARR SABER MÁS}

FAD. (2017). Centro Reina Sofía sobre Adolescencia y Juventud. http:// www.adolescenciayjuventud.org/

GonzÁlez Anleo, J. y López-Ruiz, J. A. (2017). Jóvenes Españoles "entre dos siglos" (1984-2017). Madrid: Fundación SM. Recuperado de https://www.observatoriodelajuventud.org/jovenes-espanolesentre-dos-siglos-1984-2017/

INJUVE. (2017). Observatorio del Instituto de la Juventud. http://www. injuve.es/observatorio 